\title{
QUANTUM CHEMISTRY IN MOLDOVA - 50 YEARS
}

\author{
B. Bersuker \\ Institute of Chemistry, Academy of Sciences of Moldova \& Department of Chemistry and Biochemistry, \\ The University of Texas at Austin, Austin TX, USA \\ Email: bersuker@cm.utexas.edu
}

\begin{abstract}
An overview of the main achievements of the Laboratory of Quantum Chemistry of the Institute of Chemistry of the Academy of Sciences of Moldova during the 50 year of its existence is briefly outlined. The main fundamental "non-transient" result obtained in this laboratory is the generalization of the Jahn-Teller effect theory formulated and proved as a general law of instability of polyatomic systems and its application to a variety of physical, chemical, and biological problems, including al-range spectroscopy, geometry and spectra of molecular systems, structural phase transitions, ferroelectricity, stereochemistry and crystal chemistry, chemical activation and reactivity, electron transfer in mixed-valence compounds, and electron-conformational transitions in biological systems.
\end{abstract}

\section{Introduction}

Fundamental research in quantum chemistry in Moldova started in 1959 together with the organization of the Institute of Chemistry of the Academy of Sciences of Moldova (IC ASM) (till 1961 it was the Moldavian Branch of the Academy of Sciences of the USSR). Investigations on this subject were initiated by A. V. Ablov, an internationally renowned scientist with a bright vision and deep understanding of the progressive trends in science. At that time quantum chemistry in the Soviet Union was in its embryonic stage suffering from a blow from the ruling authorities for employing the "idealistic" resonance theory. Scared by that atmosphere of ideological dogmas many Soviet chemists tried to avoid direct involvement in quantum theory of electronic structure and chemical bonding. A. V. Ablov ignored these circumstances and took the risk to invite me, a theoretician, to join him in his laboratory and to start quantum-chemical investigation of his experimental results on optical spectra and magnetic properties of transition metal compounds.

At that time I had already my doctoral thesis from Leningrad University and teaching experience from Beltsy Pedagogical Institute. Soon I got my first research student Iu. G. Titova followed later by B. G. Vekhter, S. S. Budnikov, I. Ya. Ogurtsov, S. A. Borshch, V. Z. Polinger, S. S. Stavrov, G. I. Bersuker, M. D. Kaplan, N. N. Gorinchoi, and many others (see the list in [1]). In several years we advanced to the point that in 1964, after I got my next (D. Sc.) degree and some of my coworkers got their PhD degrees, the laboratory of Quantum Chemistry (hereafter LQC) was organized within the Institute of Chemistry.

In recent years the LQC had a bad time. Transit to independence, democracy and free market deprived Republic of Moldova from its decades established sources of rough materials and consummation markets. Lack of investments and very high unemployment further deteriorated the economic situation as a whole. Funding of basic research decreased sharply to the point that salaries went to the level of poverty and the laboratory buildings were not always heated in the winter months. In these conditions many workers of the LQC left the country seeking jobs abroad. From the many Ph. D. and Dr. Hab. of LQC only a small group headed by I. Ya. Ogurtsov survived and kept the LQC alive continuing its research trends. This was possible due to a series of international grants from USA and EU. The LQC is not only alive but has very interesting results in fundamental studies of structure and properties of matter.

50 years is a fair period to make some brief summary of the achievements of this laboratory. A part of the results obtained earlier are reviewed in previous publications [2], and in Ref [1] there is list of a major part of the original publications of the LQC. What follows is an updated brief outline of the main achievements of this lab. The main scientific interests revolve around the central theme known in the literature as the Jahn-Teller Effect (JTE), its generalization being discussed briefly in the next Section. In line of the development of the theory of the JTE and pseudo JTE (PJTE) and their generalizations, numerous applications of the theory to a variety of problems in physics, chemistry, and biology were worked out. These include al-range spectroscopy, structural phase transitions, ferroelectricity, stereochemistry and crystal chemistry, chemical activation and reactivity, electron transfer in mixed-valence compounds, electronconformational transitions in biological systems, etc. Together with the doctoral theses of people from other research institutions supervised by the members of the LQC the number of $\mathrm{PhD}$ prepared in this lab counts for more than 50; 8 of them earned also the degree of "Doctor Habilitat". In view of the very large number of publications of this lab we cite here mostly some books and review articles [1-3, 6-68] that make links to the other (many hundreds of) original publications, occasionally mentioning also original works where necessary. Many of these publications are reviewed and cited in the two recent books $[1,3]$.

Fundamental achievement: general law of instability of polyatomic systems.

Before going to mention more particular achievements of the LQC I should like to briefly discuss the following question: as a result of half a century work are there any achievements of this laboratory that may be considered as 
"non-transient", meaning a fundamental, general, and rigorous result that on merits remains in science forever? In my judgment the answer to this question is yes, this laboratory has such a "global" contribution to the theory of structure of matter. This achievement can be formulated as follows: development of the concept of vibronic interactions. In the literature this trend is more often cited in its applications aspect as the Jahn-Teller Effect (JTE). As a generalization of the concept of vibronic interactions and JTE the general law of instability of polyatomic systems was formulated, proved, and confirmed by experimental data.

What is the essence of this result and why may this achievement of the laboratory be considered as fundamental and "non-transient"?

The works on these issues began at the very onset of quantum chemistry investigations at the IC ASM. At that time (1959) there were no studies of the JTE in the Soviet Union and only a couple of papers on this subject published in International journals (including the first one by H. Jahn and E. Teller [4]). The first formulation of the JTE was that nonlinear molecules in electronically degenerate states are unstable with respect to spontaneous distortions that remove the degeneracy. This simple statement was shown to lie in the base of a variety of novel effects and applications in physics, chemistry, and biology. The LQC made essential, sometimes seminal, contributions to this field partly discussed briefly in the next sections of this paper. There are many other authors contributing to this field; a review of the trend as a whole can be found in our recent book [3].

But what we claim as a more "global" achievement is the generalization of the original JTE and PJTE related to systems with exact degeneracy or near-degeneracy (quasidegeneracy) to any and all polyatomic systems in degenerate and non-degenerate states. We proved that the original JTE is just a part of a more general phenomenon that relates possible instabilities and distortions of any polyatomic (molecular or crystalline) system to the presence of two or more electronic states (degenerate or non-degenerate) that become mixed under the nuclear displacements. We formulated a general law of instability and distortions of polyatomic systems: The necessary and sufficient condition of instability and distortions of high-symmetry configurations of polyatomic systems is the presence of two or more electronic states that interact sufficiently strongly under nuclear displacements in the direction of instability. This result is most general and rigorous; it is based on first principles of quantum mechanics.

The JTE is just a particular case of this law when the energies of the two or more electronic states are degenerate or near-degenerate (in the latter case it is termed PJTE). Obviously, the number of JT systems is rather limited. The formulated above law of instability includes any system and (what is essential) excludes other sources of instability. The requirement of strong enough interaction between the electronic states limits the cases of possible distortions by the condition of the pseudo JTE, and these states are conventionally termed pseudodegenerate. Thus the formulated law of instability is indeed a global property of matter relevant to all imaginable distortions and symmetry breaking, e.g., chemical reactions, electron-conformational transitions, lattice formation and phase transitions, molecular motors and other biomechanical processes in biology, etc. All of these (and other relevant) phenomena are degeneracy (pseudodegeneracy) controlled making the law of instability a general tool of problem solving on the electronic level, as they can be treated by the extended JTE methodology [3].

An exciting development of this result followed when we found out that there is a similar conclusion in particle physics, namely, that "symmetry breaking is always associated with a degeneracy" [5] (this statement includes also the particle analog of pseudodegeneracy). Relating this result to the above law of instability of polyatomic systems we can state that Nature tends to avoid degeneracy by means of symmetry breaking. The "global" meaning of this statement may be hardly neglected.

\section{Tunneling splitting in JT systems}

One of our earlier results in the JTE theory was the prediction of the splitting of the vibrational levels of systems with the JTE. Since in many cases the JT distortions produce several equivalent minima of the APES, it occurred to us that if these minima are deep enough and there are localized states in them, there may be a special type of quantummechanical tunneling between the minima resulting in specific tunneling splitting of the vibrational levels in the minima $[1,3,7,10,11]$. If the system is prepared in one of these minima (which is usually the case), tunneling corresponds to a kind of pulse motions in which the system "jumps" from one minimum of the APES to another, equivalent, but with another direction of the JT distortion, realizing a new kind of molecular motions. This phenomenon was initially predicted in one of the first publications of the LQC in 1960, and it was later multiply conformed experimentally. Together with many important consequences this achievement (after official support by ten research institutions of the USSR) was qualified as a "Scientific Discovery" registered in the USSR in 1978 under No 202 [15]. In further development of the idea of tunneling splitting several students and coworkers of the LQC took part including V. Z. Polinger, B. G. Vekhter, I. Ya, Ogurtsov, and others.

The problem of tunneling splitting acquired a new twist in recent years in relation to the problem of the topological (Berry) phase. The latter emerged initially from the JT E®e problem where, because of the conical intersection on the APES, the electronic wavefunction is not single-valued, and to make the total wavefunction single-valued (as required by quantum mechanics) a special phase factor should be attached to the nuclear wavefunction. This additional phase 
(often called Berry phase) changes the ordering of the vibronic energy levels of the system and makes the ground state double degenerate. In our works in the 60 s the ground state emerged nondegenerate, so there was a problem that remained unexplained for many years. In several recent papers $[1,3,51]$ it was shown that if the quadratic terms of vibronic coupling are sufficiently strong, the Berry phase problem disappears as there are four conical intersections instead of one, and the ground state becomes nondegenerate in accordance with our earlier results.

\section{Origin of instability of molecules and crystals}

We already mentioned in Section 2 the general law of instability as one of the most fundamental achievements of the LQC. But the way to this result was long and laborious. The starting point was the JTE which states that highsymmetry configurations in degenerate states are unstable with regard to JT active nuclear displacements, and this effect may remain also in cases when instead of exact degeneracy of the electronic states there is near degeneracy (called pseudodegeneracy) provided the energy gap between the interacting states $\Delta$ is small enough as compared with the vibronic coupling parameter $\mathrm{F}$ and the rigidity of the system $\mathrm{K}_{0}$. The pseudo JTE was assumed to be a second order perturbation theory effect that may be significant only for very small $\Delta$ values. We started by questioning this understanding. Presenting the curvature of the APES in the direction of instability K as a sum of the primary (nonvibronic) part $\mathrm{K}_{0}$ plus the vibronic (pseudo JT) coupling to excited states $\mathrm{K}_{\mathrm{v}}, \mathrm{K}=\mathrm{K}_{0}+\mathrm{K}_{\mathrm{v}}$, we have shown $\mathrm{K}_{0}$ is always positive, $\mathrm{K}_{0}>0$, and hence a negative curvature (instability) can be obtained only due to the negative PJT contribution $\mathrm{K}_{\mathrm{v}}$ provided it is large enough, $\left|\mathrm{K}_{\mathrm{v}}\right|>\mathrm{K}_{0}[1,3,28,39]$. If only one excited state $\mid 1>$ contributes to the PJT mixing to the ground state $\mid 0>$ under nuclear displacements $\mathrm{Q}, \mathrm{K}_{\mathrm{v}}=-\mathrm{F}^{2} / \Delta$, and $\mathrm{F}=<0\left|(\partial \mathrm{H} / \partial \mathrm{Q})_{0}\right| 1>$, so we get a tool of prediction if, where, and why instabilities occur.

The meaning of this result is very significant: $\mathrm{K}_{0}>0$ means that all the instabilities of high-symmetry configuration of any polyatomic system are due to and only to the pseudo JTE. We have also shown that from the interatomic bonding point of view the distortions occur due to the formation of additional covalency between the atoms via the overlap of their orbitals that is zero in the high-symmetry configuration. We used these ideas of instability and distortions to reveal the origin of a variety of phenomena and performed many electronic structure calculations; all of them confirmed numerically the theoretical predictions $[1,3,22,39,52,57]$. Section 2 above and several Sections below discuss some of these results. Most of the works on this topic were performed by V. Z. Polinger, N. N. Gorinchoi, I. Ya. Ogurtsov, F. Chimpoesu, O. V. Yaltichenko and others.

\section{Origin of ferroelectricity. Cooperative JTE.}

Works on the PJTE problem brought us in 1964 to the understanding that structural phase transitions in dielectric crystals should be of the same PJT origin $[1,3,8,16,44]$. While the cooperative JTE in crystals with JT centers was already revealed at the time, the cooperative PJTE was entirely unknown as the very possibility of a strong local PJTE was not well known and practically ignored.

In the best known ferroelectric crystal $\mathrm{BaTiO}_{3}$, starting with the ionic presentation, the $\mathrm{Ti}^{4+}$ ion in the highsymmetry configuration is in the center of the octahedron of $\mathrm{O}^{2-}$ ions, and the ground state of the $\operatorname{local} \mathrm{TiO}_{6}^{8-}$ cluster is a $\mathrm{A}_{1 \mathrm{~g}}$ term formed by occupied linear combinations of the oxygen atomic $2 p$ functions, while the first excited term $\mathrm{T}_{1 \mathrm{u}}$ is formed by an one-electron excitation from a $t_{1 \mathrm{u}}$ molecular orbital to the unoccupied $t_{2 \mathrm{~g}}$ combinations of the $3 \mathrm{~d}_{\mathrm{xy}}$ type atomic functions of the $\mathrm{Ti}^{4+}$ ion. We succeeded to show that the PJTE $\left(\mathrm{A}_{1 \mathrm{~g}}+\mathrm{T}_{1 \mathrm{u}}\right) \otimes t_{1 \mathrm{u}}$ results in instability of the centro-symmetric position of the titanium ion, its displacements producing a local dipole moment, and the cooperative interactions of these dipoles leads to the spontaneous polarization of the crystal [8, 44].

The APES for the $\mathrm{Ti}^{4+}$ ion has eight equivalent minimum positions at which the ion is displaced along the trigonal axes of the oxygen octahedron, and the energy barriers between them form two types of saddle points which can be thermally overcome. In this picture all the qualitative properties of the $\mathrm{BaTiO}_{3}$ ferroelectric were reproduced including the three phase transitions between the four phases (three ferroelectric phases and the para-phase), the direction of polarization in each of them, and their relative temperatures of occurrence.

The most exciting result was the prediction that only the lowest rhombohedral phase is fully ordered, the other three ones being partially disordered, meaning ordered in some direction and disordered in others. In particular, the cubic para-phase (which is macroscopically not polarized) was predicted to be fully disordered, formed by the thermal averaged positions of the $\mathrm{Ti}^{4+}$ ion over the eight equivalent minima. All the predictions of the theory were afterwards multiply confirmed by a variety of experimental data $[1,3,44]$. It is especially suggestive that the PJTE is practically independent of temperature, and hence the $\mathrm{Ti}^{4+}$ ion should be displaced along one of the trigonal axis (toward three oxygen atoms) in all the phases. This effect is seen explicitly in all the experimental direct measurements. No existing and dominating at the time theories of displacive phase transitions can explain this phenomenon. The works on this subject were realized by B. G. Vekhter, A. A. Muzalevskii, V. P. Zenchenko.

Independently, a series of investigations on the cooperative JTE and structural phase transitions with several applications were published and reviewed in a book on this subject by M. D. Kaplan and B. G. Vekhter [45]. 


\section{Multimode and multicenter JTE. Mixed-valence compounds}

Our LQC attacked the JTE problem from different sides and at different levels. In particular, we treated one of the most difficult JTE problems when there are more that one JT active modes of given symmetry which may take place already when there are more then three atoms in the system, and the number of active modes increases with the number of atoms tending to infinite for active centers in crystals. The latter case is especially important for JT impurity centers in crystals meaning center with a JT or pseudo JT effect. In a series of papers several aspects of this problem were investigated including the influence of the multimode JTE on the vibrational spectrum in infrared and Raman spectroscopy, as well as in ESR spectra [3, 22, 39]. Off-center impurities were also considered in this multimode aspect $[19,30,39]$. Works on these issues were performed by V. Z, Polinger, G. I. Bersuker, and A. Solonenko.

The multicenter JTE is obviously more difficult that the usual one-center one as it includes also the interaction between the centers, but it is also much more complicated than the infinite-center one in crystals where the symmetry of translation essentially and cooperative treatment simplifies the problem. In the QC lab the two-center, tri-center, and four-center problems were successfully solved and a variety of properties predicted, in particular, magnetic properties $[24,39]$. Most contributions to this work are due to V. Z. Polinger, L. V. Chibotaru, M. L. Rafalovich, and S. A. Borshch.

In some overlap with the multi-center problem is the one of mixed-valence compounds. The QC laboratory was one of a few in the world to develop the theory of vibronic coupling implication in these systems [1, 3, 23, 42]. For both the general case and specific systems the relation between vibronic coupling constants and the rate of electron transfer between the mixed-valence centers in view of the localization-delocalization alternative was elucidated and their more specific pseudo JTE influence on this process was investigated in view of their optical and magnetic properties. In particular, for tri-center systems the phenomenon of coexistence of localized and delocalized states was revealed for the first time. A similar effect was found in four-center systems. The problem of multi-level centers and the so-called doubleexchange interaction was also advanced significantly. As model systems, for some aspects of the problem tri-center and four-center ferredoxins were studied [1, 3, 42]. Works on these issues were performed by S. A. Borshch, I. N. Kotov, L. V. Chibotaru, I. Ya. Ogurtsov, and B. S. Tsukerblat.

\section{Chemical activation and reactivity}

Among the many chemical and biological applications of the JTE carried out in our lab chemical activation by coordination occupies a special place. We succeeded to reveal the intimate mechanism on why and how the weak interaction of a small molecule with the coordination center of a catalyst or enzyme results in its becoming more chemically active (acquiring larger reactivity) in interaction with other species, and we obtained analytical formulas for estimation of the reduction of the activation energy in such catalyst enhanced reactions $[1,3,32,57]$. The basic idea of our approach is based on the understanding that the main effect of coordination is orbital charge transfer, and that the bondings in the coordinated molecule is weakened by charge transfer to its antibonding molecular orbitals (MO) or from the bonding MO's realized via the vibronic coupling of these MO's to the nuclear framework. The solution of this problem was essentially simplified by using the introduced earlier in our lab orbital vibronic constants [22] which are now used also in a variety of other problems. We applied the theory of vibronic activation by coordination to quite a number of activation problems, mostly to activation of diatomics by transition metal systems including biological systems (e. g., activation of oxygen by hemoproteins [38]). Contribution to this part of works are due mostly to S. S Budnikov, S. P. Svitin, I.Ogurtsov, N. N. Gorinchoi, I. Balan, V.Mirzac, and others.

Most related to the catalyst problem is the activation of a molecule by the JTE or pseudo JTE. It was shown that these effects lower the activation energy of reactions that proceed in the direction of JT or pseudo JT active coordinates. The latter are determined by the degeneracy of the electronic state in the presence of the JTE or by the symmetry of the active excited state in case of pseudo JTE $[1,3,48]$.

\section{Applications to biological systems}

In application of the vibronic theory molecular systems we focused our attention also to active centers in biological systems. Transition metal centers and other prosthetic groups are of special interest in application of the JTE and the pseudo JTE for two reasons: (1) they possess degenerate or sufficiently close electronic energy levels and (2) their environment is relatively very soft to distortions (they have size dependent soft modes). Our treatment of several biological problems with the vibronic theory confirmed these statements $[1,3,38]$.

One of these problems was the origin of the trigger mechanism of hemoglobin oxygenation. It was shown earlier that the hemoglobin's fast absorption of oxygen in an oxygen environment and its release of the oxygen in nooxygen environment is due, respectively, to the out-of-plan (without oxygen) and in-plan (with coordinated oxygen) positions of the iron atom with respect to the porphyrin ring in the hemoprotein center, and the in-plan motion triggers electron-conformational changes resulting in the four-center cooperative absorption oxygen in the former case and its release in the latter one. We proved that the out-of plane displacement of the iron atom is due to the pseudo JTE, and its in-plan return by oxygenation is due to the quenching of this effect by coordination of the oxygen molecule [38]. 
This electronic mechanism improves our understanding of biological processes and provide us with tools to control them.

In the case of cytochrome $\mathrm{P}-450$ which has a similar hemoprotein as an active center oxygen coordination produces the same quenching the pseudo JTE and the iron atom moving in-plan, but because of another environment this iron atom motion results in a breakdown of a hydrogen bond and an excess electron occupying an antibonding orbital of the oxygen molecule thus strongly weakening the $\mathrm{O}-\mathrm{O}$ bonding.

The pseudo JTE is also instrumental in explaining the geometries of small ligand coordination to hemoproteins and their behavior in flash photolysis [38]. Most of the works on this subject is due to S. S. Stavrov and I. P. Dicusar.

\section{Hidden JTE}

Further developments of the JTE theory lead us to the so-called hidden JTE. We have shown that when molecular systems are distorted, but there are no apparent degeneracies or close in energy states to claim pseudo JT interaction the JTE are "hidden" in the excited states of the undistorted configuration, even when the energy gap to these states is very large. For the example of the ozone molecule (which has no degenerate ground state, neither in the distorted obtusetriangular nor in the undistorted regular triangular configurations, and it has no low-lying excited states) the distortion is shown to emerge from the JTE in the excited $E$ state situated at $\sim 8.3 \mathrm{eV}$ above the ground state. The JT stabilization energy is more than $\sim 9 \mathrm{eV}$ which makes the distorted configuration the lowest in energy [60,63]. For molecular systems with half-closed-shell electronic configurations $e^{2}$ and $t^{3}$ (formed by degenerate orbitals $e$ and $t$ ), which produce totally symmetric charge distribution and are not subject to the JTE, distortions were shown to occur due to a strong pseudo JT mixing of two excited states. Again, the pseudo JT stabilization energy in these cases is larger than the energy gap to the ground state, and the distortion is accompanied by orbital disproportionation. In some systems this produces two coexisting states, low-spin distorted and high-spin undistorted, and a novel phenomenon: JT-induced spin-crossover. Distinguished from the known spin-crossover in transition metal compounds the JT-induced spin-crossover takes place in a large variety of molecular systems from different classes including organic compounds, and it has a much lower rate of relaxation between the two states; this makes them candidates for single-molecule materials for electronic devices. $\mathrm{Ab}$ initio calculations of the hidden JTE were performed on a series of molecular systems from different classes [62, 64, 65]. These works were carried out in cooperation with the Theoretical and Computational Chemistry group of the University of Texas at Austin.

\section{Theory of physical methods of investigation}

Quite a number of works were devoted to the theory of a variety of physical methods, especially for systems with the JTE. These include first of all spectroscopy in all ranges of wavelengths including band shapes and zero-phonon lines of optical absorption and spectroscopy, birefringence, infrared and Raman spectra, ESR, gamma-resonance (Mossbauer) spectroscopy, as well as magnetic susceptibility, diffraction of X-rays and electrons, and others $[18,21,29,31,33,36$, 40]. Many members of the LQC contributed to this trend including B. G. Vekhter, I. Ya. Ogurtsov, V. Z. Polinger, S. A. Borshch, L. A. Kazantseva, V.L. Ostrovskii, V.P. Khlopin, Iu. V. Shaparev, and Iu. E. Perlin.

\section{Electronic structure calculations and molecular modelling}

In the earlier years of existence the QC laboratory had no sufficient computers for electronic structure calculations, so the earlier works with such calculations were performed using analytical methods and semiempirical calculations and more emphasize was put on novel qualitative results and their physical meaning. For quite a number of small to moderate molecular systems electronic structure calculations were used to reveal the origin of the chemical bonding, reactivity, and spectra $[12,48]$. In further development of the semiempirical methods some improvements were introduced and appropriate computer programs worked out. In particular, a method of fragmentary calculations was suggested [53]. Of particular interest was the semiempirical quasirelativistic approach which happened to be the first application of relativistic theory to MO LCAO calculations [14]. Ab initio methods were worked and applied to calculations of the PJTE $[28,55]$, as well as hidden JTE and origin of distortions of linear molecules [62, 65], and to evaluate important chemical processes, e.g., to reveal the mechanism of activation of oxygen on transition metal coordination systems [59, 60]. These works were carried out with Iu. G. Titova, S. S. Budnikov, B. A. Leizerov, M. A. Baraga, A. S. Dimoglo, S. M. Bardina, F. A. Spataru, I.Ya.Ogurtsov, L. Baciu, N. N. Gorinchoi, and V. Z. Polinger.

In more recent years a method combined quantum-classical calculations of large metallobiochemical systems was worked out and applied to specific calculations in collaboration with the Theoretical and Computational Chemistry group of the University of Texas at Austin. In this method the active site is calculated in the usual quantum-chemical (QM) approximations, whereas the larger (biological) part is evaluated by means of molecular modelling (MM) with a special interface between them. Distinguished from other QM/MM methods worked out earlier by other authors, our method takes into account the charge transfer (CT) between the QM and MM parts which is most important when the QM part contains transition metals. Our QM/MM/CT method is the only one that is able to take into account this charge transfer [53]. 


\section{Stereochemistry and crystal chemistry}

Obviously, the JTE has direct influence on the formation of molecular shapes, crystal lattices, and other properties of polyatomic systems controlled by the electronic structure. A variety of such problems were considered in QC laboratory among which we mention here the phenomenon of plasticity of the coordination sphere around some transition metals, formation of distortion isomers, and the origin of the active and inert lone pairs $[1,3,13]$. The plasticity effect came out to be very useful in understanding the origin of different coordination symmetries for the same transition metal (mostly $\mathrm{Cu}(\mathrm{II}))$ in the same first coordination sphere. We have shown that such differences take place due to the JTE which makes the coordination sphere "soft" to the influence of the rest of the lattice. The publication on this effect [13] got a record number of references (more than 500).

\section{A method of computer-based prediction, screening, and design of biological activity}

In a more practical aspect, the so-called electron-conformational (EC) method of computer-based prediction of biological activity in drug design and toxicology was worked out in the LQC and applied to a large number of relevant problems $[41,56,68]$. Distinguished from other methods with the same goal known mostly as quantitative structureactivity relationships (QSAR), the EC method uses the quantum-chemical calculated electronic structure of conformations as the unique descriptor of their activity in the interaction with the bioreceptor and does not employ statistics at the stage of pharmacophore and toxicophore evaluation, thus making the results most reliable (within the accuracy and completeness of the training set). Supplemented with an empirical parameterization of the anti-pharmacophore (and other) influence of out-of-pharmacophore groups, the EC method yields predictions of the activity quantitatively, while the pharmacophore (or toxicophore) itself, presented in the form of a numerical matrix of electronic structure parameters, can be conveniently used also for screening new compounds with respect to the activity under consideration. Works on this topic were carried out by A. S. Dimoglo, M. Iu. Gorbachov, I. N. Choban, I. Ya. Ogurtsov, N. N. Gorinchoi,

\section{References}

[1]. The Jahn-Teller Effect and Beyond. Selected Works of Isaac Bersuker with Commentaries, Eds. J. E. Boggs and V. Z. Polinger, Acad. Sc. Moldova \& Univ. Texas Austin, 2008.

[2]. Bersuker I. B., Quantum chemistry in the institute of chemistry for 20 years, Proc. Acad. Sci., MoSSR, Ser. biol. chem. nauk, N 5, $42-52$ (1979); Quantum chemistry in Moldova, Khim. Fiz., 11, N5, 588-594 (1992).

[3]. Bersuker I. B., The Jahn-Teller Effect, Cambridge University Press, Cambridge, 2006.

[4]. Jahn H. and Teller E., Proc. Roy. Soc .London, A 161, 220 (1937)

[5]. Weinberg S., Quantum Theory of Fields, Cambridge University Press, Cambridge (UK), 1995, Chap. 11

[6]. Bersuker I. B. and Ablov A. V., Chemical Bonds in Complex Compounds, Academy of Sciences, MoSSR, Kishinev, 1962, 208 p.

[7]. Bersuker I. B., Zh. Eksp. Teor. Fiz., 43, N 4, 1315-1322 (1962) (Sov.Phys.-JETP 16, N 4, 933-938, (1963)).

[8]. Bersuker I. B., Phys. Lett. A, 20, N 6, 589-590 (1966).

[9]. Bersuker I. B. and Polinger V. Z., Zh. Eksp. Teor. Fiz. (Sov. Phys-JETP), 66, N 6, 2078-2091 (1974).

[10]. Bersuker I. B., Vekhter B. G. and Ogurtsov I. Ya., Uspekhi Fiz. Nauk (Sov. Phys.-Uspechi), v.116, N 4, 605-641 (1975).

[11]. Bersuker I. B., Coord. Chem. Revs., 14, N 4, 357- 412 (1975).

[12]. Bersuker I. B., Electronic Structure and Properties of Coordination Compounds. Introduction to the Theory (Russ.), Second Edition, Chemistry, Leningrad, 1976, 350 p.

[13]. Gazo J., Bersuker I. B., Garaj J., Kabesova M., Kohout J., Langfelderova H., Melnik M., Serator M., Valach F., Coord. Chem. Revs., v.19, N 3, 253- 297 (1976).

[14]. Bersuker I. B., Budnikov S. S. and Leizerov B. A., Internat. J. Quant. Chem., 11, N4, 543-559, (1977).

[15]. Bersuker I. B., Scientific Discovery: "The Phenomenon of Tunneling Splitting of Energy Levels of Polyatomic Systems in Electronic Degenerate States", State Registered, USSR, 1978, with the priority from September 12, 1960. [Diploma N 202 issued in 1979] Official Bulletin of the State Committee on Inventions and Discoveries, N 40, October 1978.

[16]. Bersuker I. B. and Vekhter B. G., Ferroelectrics, 19, N 3-4, 137-150 (1978).

[17]. Ogurtsov I.Ya., Shaparev Yu.V., Bersuker I.B., Opt. Spectrosc., 45, N 4, 672 (1978).

[18]. Ogurtsov I.Ya. and Kazantseva L. A., J. Molec. Struct., 55, 301-307 (1979).

[19]. Bersuker G. I., Polinger V. Z. and Khlopin V. P., Fiz. Tverdogo Tela, 24, 2471-2476 (1982).

[20]. Bersuker I. B. and Polinger V. Z., Vibronnie Vzaimodeistvia v Molekulakh i Kristallakh (Russ.), Nauka, Moscow, $1983,336 \mathrm{p}$.

[21]. Ogurtsov I.Ya., Ostrovskii V.L., Bersuker I.B., Opt. Spectrosc., 53, N2, 356 (1982); Mol. Phys., 48, N 1, 13 (1983); 50, N 2, 315 (1983). 
[22]. Bersuker I. B., The Jahn-Teller Effect and Vibronic Interactions in Modern Chemistry, Plenum Press, New York, 1984, 320 p.

[23]. Borshch S. A., Kotov I. N., and Bersuker I. B., Chem. Phys. Lett. 3, N 3, 264-270 (1984); Khim. Fiz., 3 , № 5. 667-671 (1984).

[24]. Polinger V. Z., Chibotaru L. F.and Bersuker I. B., Mol. Phys. 52, N 6, 1271-1289 (1984).

[25]. Vekhter B. G. and Kaplan M. D., J. Exp. Teor. Phys., 87, N 5 (II), 1774-1783 (1984).

[26]. The Jahn-Teller Effect. A Bibliographic Review, Ed. I.B. Bersuker, IFI/Plenum, New York, 1984, 590p.

[27]. Bersuker I. B. and Polinger V. Z., in: The Jahn-Teller Effect in Localized States, Eds. Iu.E.Perlin, M.Wagner, North-Holland, Amsterdam, 1984, p.21-85.

[28]. Bersuker I. B., Gorinchoy N. N. and Polinger V. Z., Theor. Chim. Acta, 66, N 1, 161-172 (1984).

[29]. Ogurtsov I.Ya., Optics Spectrosc., 56, N 1 (1984) 60.

[30]. Bersuker G. I. and Pollinger V. Z., Phys. Status Solidi B, 125, 401-408 (1984); Fiz. Tverdogo Tela, 26, $2549-2551$ (1984); Chem. Phys. 86, 57-66 (1984).

[31]. Ogurtsov I.Ya., Zh. struct. Khim, 26, N 2 (1985) 46; I.Ya.Ogurtsov, V.L.Ostrovski, Mol. Phys., 54, N 1 (1985) 119 .

[32]. I. B. Bersuker, S. S. Budnikov, In: Fundamental Research in Homogeneous Catalysis, Ed. A. E. Shilov, Gordon and Breach, London, v.2, 1986, p.557-568.

[33]. I. B. Bersuker and I. Ya. Ogurtsov, Adv. Quant. Chem., v.18, Acad. Press, N. Y., 1986, p.1-84.

[34]. I. B. Bersuker, Uspekhi Khimii, v.55, N 7, 1986, p.1057-1082 (Russ. Chem. Revs., v.55, 1986, p.581-596).

[35]. I. B. Bersuker, Electronic Structure and Properties of Coordination Compounds, Third Edition, Chemistry, Leningrad, 1986, 288 p.

[36]. I.Ya. Ogurtsov, Zh. Struct. Khim., 27, N 3 (1986) 65.

[37]. I. B. Bersuker, Effect Yana-Tellera i Vibronnye Vzaimodeistviya v Sovremennoi Khimii (Russ.), Nauka, Moscow, $1987,344 \mathrm{p}$.

[38]. I. B. Bersuker and S. S. Stavrov, Coord. Chem. Revs, 88, 1-68 (1988).

[39]. I. B. Bersuker and V. Z. Polinger, Vibronic Interactions in Molecules and Crystals, Springer-Verlag, BerlinHeidelberg-New York, 1989, 422 p.

[40]. I.Ya.Ogurtsov, Optics Spectrosc., 67, N 6 (1989) 1251.

[41]. I. B. Bersuker and A. S. Dimoglo, in: Rev. Comput. Chem.y, v.2, Eds. K.B.Lipkowitz and D.B.Boyd, VCH, New York, 1991, pp 423-460.

[42]. I. B. Bersuker and S. A. Borshch, Adv. Chem. Phys. 1992, v. 81, pp 703-782.

[43]. I.Ya. Ogurtsov, New J. Chem., 17, N 1-2 (1993) 137.

[44]. I. B. Bersuker, Ferroelectrics, 164, 75-100 (1995).

[45]. M. D. Kaplan and B. G. Vekhter, Cooperative Phenomena in Jahn-Teller Crystals, Plenum Press, New York, 1995.

[46]. I.Ya.Ogurtsov, N.N.Gorinchoi, J. Mol. Struct. (Theochem.), 331, 281-287 (1995).

[47]. I. B. Bersuker, J. Coord. Chem., 34, 289-338 (1995).

[48]. I. B. Bersuker, Electronic Structure and Properties of Coordination Compounds. Introduction to the Theory, Wiley, New York, 1996, pp xxiv +668 .

[49]. I. B. Bersuker, J. Comput. Chem., 18, 260-267 (1997).

[50]. I. B. Bersuker, M. K. Leong, J. E. Boggs and R. S. Pearlman, in: Combined Quantum and Molecular Mechanics Methods, Eds. J. Gao and M. Thompson, ACS Symp. Series 712, 1998, pp. 66-91.

[51]. H. Koizumi , I. B. Bersuker, Phys. Rev. Lett., 83, 3009-3012 (1999).

[52]. Ogurtsov I.Ya., G. Munteanu, L. Bantush, I.B. Bersuker, J. Mol. Struct. (THEOCHEM), 541, 141-148 (2001).

[53]. Bersuker I. B., in Computational Chemistry: Reviews of Current Trends, V. 6, Ed. J. Lesczinski, World Scientific, Singapore, 2001, pp 69-135.

[54]. Bersuker I. B., Chemical Reviews, 101, 967-1114 (2001).

[55]. Bersuker I. B., Balabanov N. B., Pekker D. M. and Boggs J. E., J. Chem. Phys., 117, 10478-10486 (2002).

[56]. Bersuker I. B., Current Pharmaceutical Design, 9, 1775-1606 (2003).

[57]. Ogurtsov I.Ya., Mirzac V., Adv. Quant. Chem., 44, 401-412 (2003).

[58]. Bersuker I. B., Adv. Quant. Chem., 44, 1-12 (2003).

[59]. Ogurtsov I.Ya., Mirzac V., Bersuker I.B. Proceedings of the $28^{\text {th }}$ Congress of American - Romanian Academy of Sciences and Arts, June 3 - 9, 2003, Targu - Jiu, Romania, pp.979 - 982; Ogurtsov I.Ya., Gorincioi N., Baciu L., Bersuker I. B., ibid., pp. $987-990$.

[60]. Ogurtsov I.Ya., Gorinchoy N., Turta C.I., Bersuker I. B., Baciu L. Annals of Vest University of Timisoara 
(Romania), 12(3), 987 - 990 ( 2003) Ogurtsov I.Ya., Gorincioi N., Baciu L., Bersuker I. B., ibid., 12(3), 12271232 (2003).

[61]. Bersuker I. B., In: Fundamental World of Quantum Chemistry, V. 3, Eds. E. J. Brandas and E. S. Kryachko, Kluwer, Dordrecht, 2004, pp 257-271.

[62]. Garcia-Fernandez P., Bersuker I. B. and Boggs J. E., Phys. Rev. Lett., 96, 163005 (2006); J. Chem. Phys., 125, 104102 (2006).

[63]. Ogurtsov I.Ya., Balan I., Munteanu G., Int. J. Quant. Chem., 106(6), 1413-1418 (2006).

[64]. Ogurtsov I., Gorinchoy N., Balan I., J. Mol. Struct., 838,107-111 (2007).

[65]. Bersuker I. B., in Proceedings of the XIX Symposium on the Jahn-Teller Effect (Trieste, Italy, 2006), J. Mol. Struct. 838, 44-52 (2007).

[66]. Zou W., Bersuker I. B. and Boggs J. E., J. Chem. Phys. 129, 114107 (2008).

[67]. Gorinchoy N.N., Ogurtsov I.Ya. and Arsene I., Chem. J. Moldova, 3, 105-111 (2008).

[68]. Gorinchoy N.N., Ogurtsov I. Ya., Tihonovschi A., Balan I., Bersuker I. B., Marenich A., and Boggs J. E., Chem. J. Moldova, 3, 94-104 (2008). 